Tome 37, no 3. - Mars 1930.

RÉSultats DE LA

Mission scientifiQue suisse en Angola, 1928-1929.

\title{
Formicides de l'Angola
}

par le

\section{$D^{r}$ F. SANTSCHI}

Avec 10 figures dans le texte.

\section{INTRODUCTION.}

Les Fourmis qui font l'objet de cette étude ont été récoltées par M. le Docteur A. Monard au cours d'une mission, de septembre 1928 à fin janvier 1929, dans la région du Rio Kubango, au sud de l'Angola. Bien que fort occupé, M. Monard a rapporté une intéressante collection où sont représentées 5 sousfamilles, 24 genres, dont 16 sous-genres sont distincts et un nouveau, 53 espèces dont 19 races ou sous-espèces ou stirps et 28 variétés, soit 73 formes différentes. Dans ce nombre il y a 5 espèces, 4 races et 8 variétés qui sont nouvelles.

Or, les Fourmis de l'Angola ont été étudiées par Forel et moimême dans cinq publications dont voici un court résumé.

1901. Forel. Einige neue Ameisen aus Süd-Brasilien, Java, Natal und Mossamedes. Description de 8 espèces, 2 races et 3 variétés.

1910. Forel. Note sur quelques fourmis d'Afrique. Cite 7 formes dont 2 espèces, 2 races et 1 variété sont nouvelles.

1909. Forel. Fourmis du Benguela récoltées par C. Wellman. Cite 15 formes différentes dont 5 espèces, et 3 variétés sont nouvelles.

1911. Santschi. Nouvelles fourmis du Congo et du Benguela. Je décris ici 2 espèces, 5 races et 3 varités nouvelles et cite 5 autres formes, toutes reçues de mon regretté ami Jean Cruchet. J'ai revu ou décrit quelques autres formes, reçues du même, dans diverses publications dont la liste se trouve au chapitre de la Bibliographie.

1925. Santschi. Mission de Rohan-Chabot en Angola, Formicides. Les spécimens récoltés par cette mission comprennent 33 formes

Rev. Suisse de Zool. T. 37. 1930. 
différentes sans compter quelques ô et $q$ indéterminables. Ces 33 formes appartiennent à 27 espèces dont 2 sont nouvelles; il y a en outre 1 race et 2 variétés nouvelles.

\section{LISTE DES ESPÈCES, RACES ET VARIÉTÉS TROUVÉES JUSQU'ICI DANS L'ANGOLA.}

Celles qui sont nouvelles pour l'Angola et rapportées par le $D^{r}$ Monard sont précédées d'une *.

\section{Ponerinae}

* Centromyrmex constanciae Arnold.

Platythyrea crucheti Sants.

) lamellosa Rog. st. suturalis For.

") cyriluli For.

* Plectroctena mandibularis $\mathrm{Sm}$.

) dentata Sants.

Paltothyreus tarsatus F. v. medianus Sants.
))
") v. delagoensis Em.

Megaloponera foetens F. v. crassicornis Gerst.

Bothroponera soror Em.

picardi For.

Ophtalmopone hottentota For. mocquerysi Em.

*Odontomachus haematodes $\mathrm{L}$.

\section{Dorylinae}

*Dorylus (Dorylus) moestus Em. v. claripennis Sants.

Dorylus (Anomma) nigricans Ill. st. molestus Gerst.

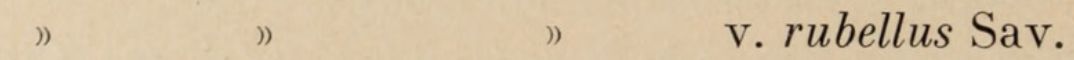

Dorylus (Rhogmus) fimbriatus Schuc.

kohli Wasm. v. minor Sants.

\section{Pseudomýrinae}

*Tetraponera ophtalmica Em. st. angolensis n. st.

\section{Myrmicinae}

* Myrmicaria natalensis Sm. 
* Myrmicaria opaciventris Em.

$\begin{array}{lll}" \text { " } & \text { v. crucheti } \text { Sants. } \\ \text { " } & \text { " } & \text { v. congolensis For. } \\ \text { " } & \text { baumi For. } & \end{array}$

Messor capensis Mayr v. tropicorum For.

Pheidole megacephala F.

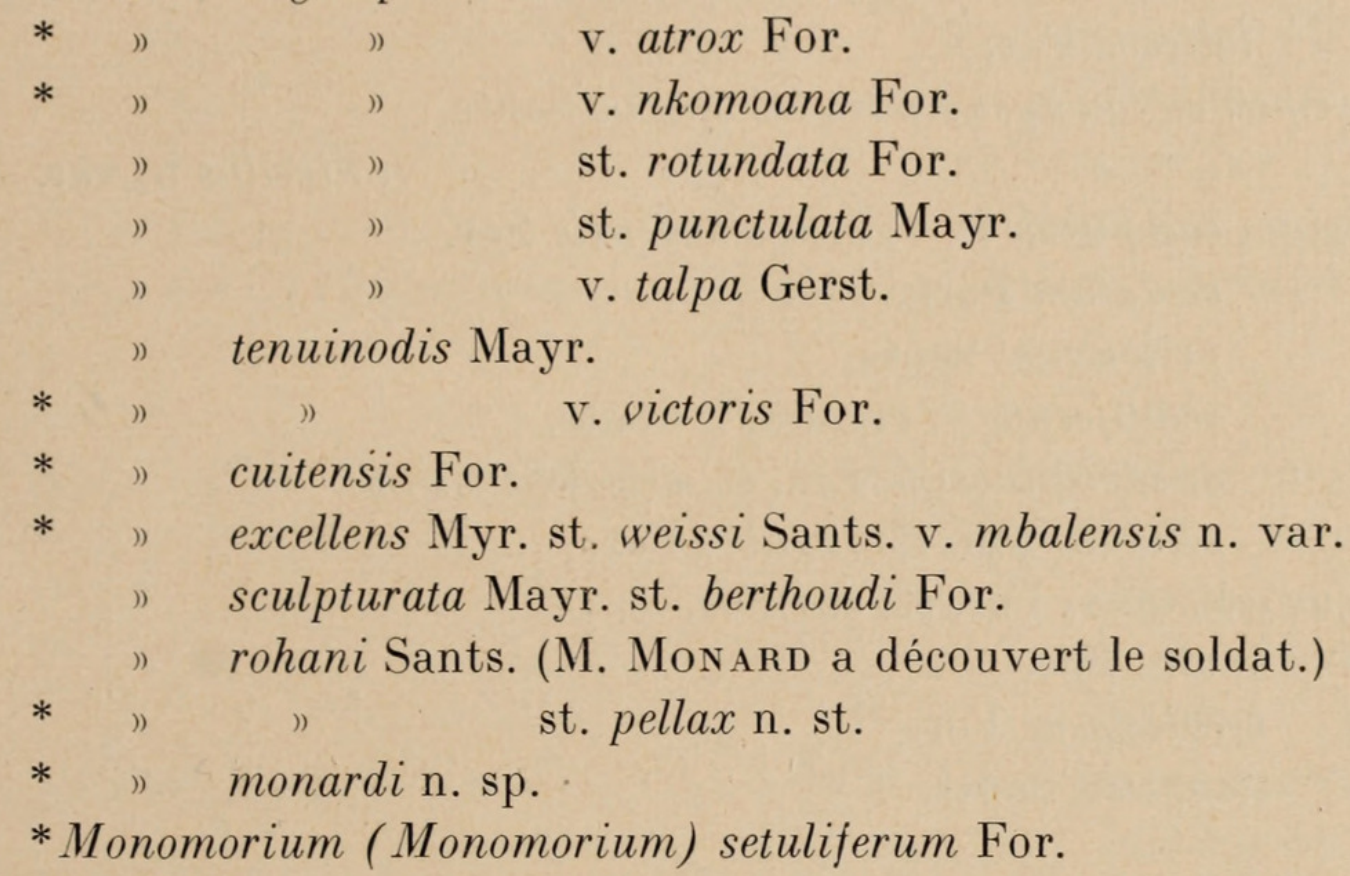
v. latior Sants.
(Xeromyrmex) salomonis L. junodi For. v. opacior For.

* Cardiocondyla (Loncyda) n. sg. monardi n. sp.

Solenopsis punctaticeps Mayr.

Oligomyrmex angolensis Sants.

Crematogaster (Atopogyne) buchneri For.

clariventris Mayr v. bimpressa Mayr. (Sphaerocrema) amita For.

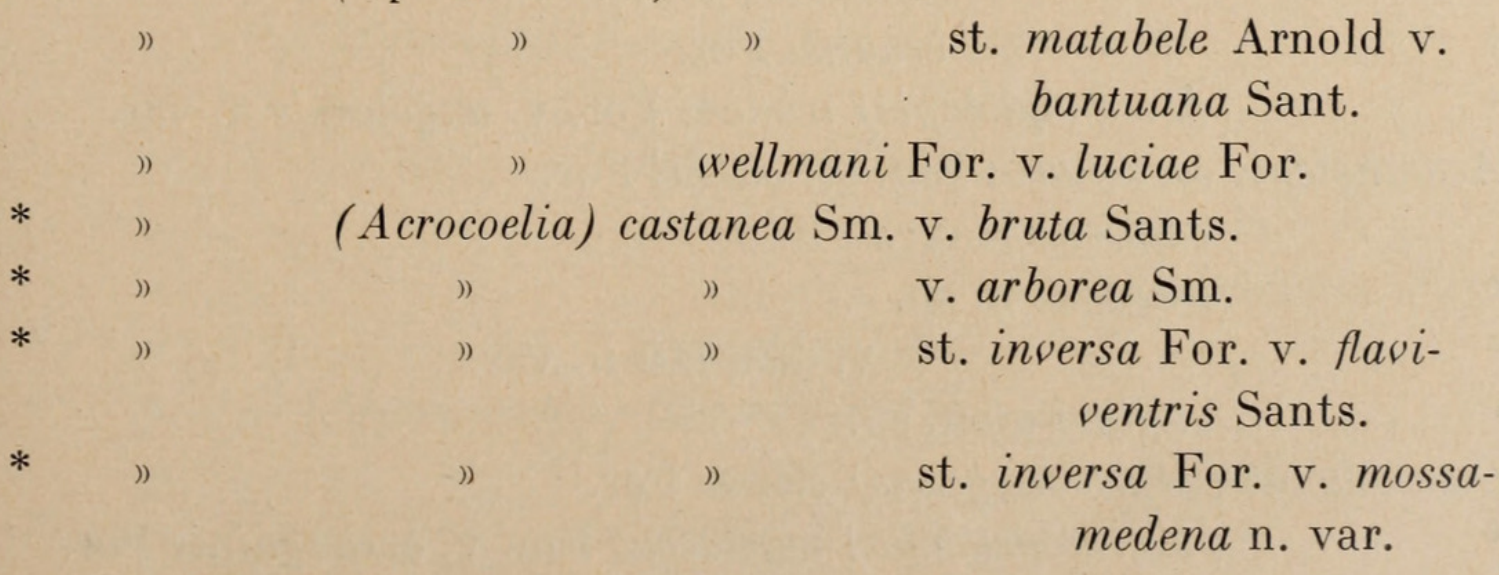


*Crematogaster (Acrocoelia) gerstaeckeri D.T. st. bulawayensis For. Ocymyrmex picardi For.

$\begin{array}{lll}\text { * } & \text { weitzeckeri } \text { Em. st. fortior Sants. } \\ \text { \# } & \text { ") } & \text { st. transversus } \text { Sants. } \\ & \text { " } & \text { st. monardi } \text { n. st. }\end{array}$

Atopomyrmex mocquerysi André st. opacus Sants.

* ” " " v. nigellus $\mathrm{n}$. var.

Tetramorium caespitum L. v. mossamedensis For.

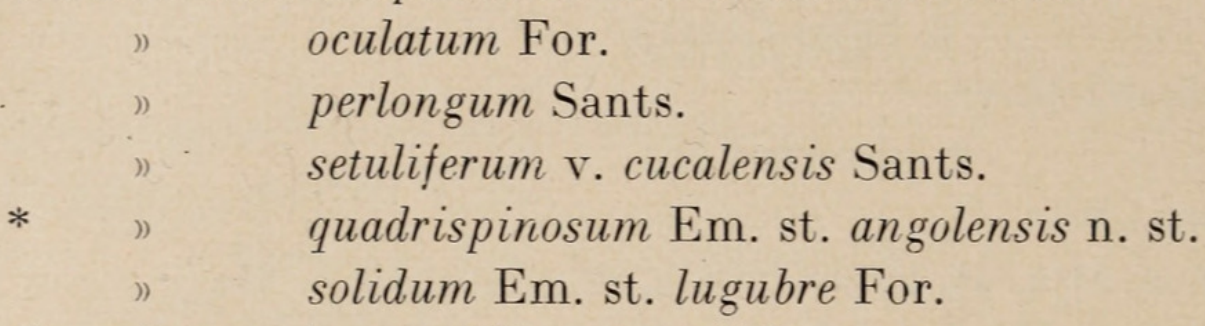

Cataulacus wismanni For. st. otii For.

baumi For.

brevisetosus For.

elongatus Sants.

\section{Dolichoderinae}

Tapinoma (Ecphorella) wellmani For.

Technomyrmex brevicornis Sants. (in lit.).

\section{Formicinae}

Plagiolepis (Anacantholepis) van der kelleni For.

* " " fuscula Em.

Anoplolepis (Zealleyella) custodiens Sm.

steingroveri For.

* „ " braunsi For.

* „) (Mesanoplolepis) trimeni For. v. angolensis n. var.

Acantholepis carbonaria Em. st. baumi For.

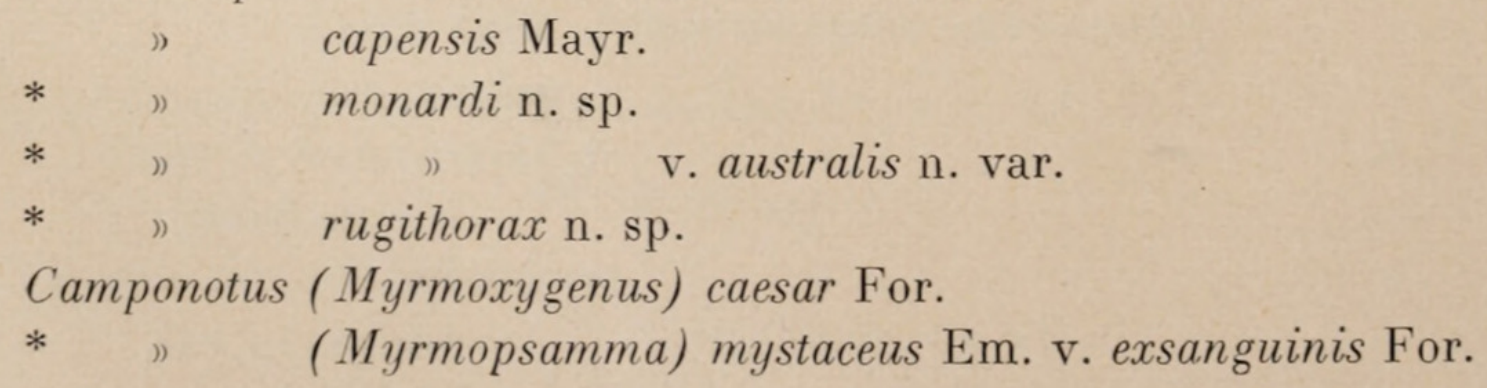


*Camponotus (Myrmoturba) maculatus F. atramentarius For. v. cluis For.

st. radamoides For. v. liocnemis Em.

st. radamoides v. calceatus n. var.

st. pictiventris Mayr.

st. cavalus Sants.

st. manzer For.

ballioni Em. v. boera For.

(Myrmosericus) rufoglaucus Jerd. st. cinctellus Gerst.

)

)

))

))

vestitus $\mathrm{Sm}$. v. in-

tuens For.

st. flasomarginatus

Mayr v. pausipubens Sants.

st. controversus

Sants.

st. contruversus V. chaboti Sants.

"
$"$
"
(Tanaemyrmex)

)
)

)) eugeniae For.

v. pectitus Sants.

v. lujai Sants (in lit.).

st. bombycinus $\mathrm{n}$. st.

(Myrmosaga) schoutedeni For.

(Myrmopiromis) fulsopilosus Gerst. v. flasopilosus Em.

(Myrmopelta) vividus $\mathrm{Sm}$. v. reginae For.

v. meinerti For. chrysurus Gerst.. 
Camponotus (Myrmotrema) olivieri For. st. concordia Sants.

$\begin{array}{ll} & \text { ) } \\ * & \text { ) } \\ * & \text { ) } \\ * & \text { ) } \\ * & \text { ) } \\ * & \text { ) } \\ * & \text { ) }\end{array}$
perrisi For.

Phasmomyrmex buchneri For. crucheti Sants. avius Sants. auropubens For. v. argentopubens For. grandidieri For. st. benguelensis Sants. monardi n. sp.

(Orthonotomyrmex) sericeus F. v. sulgeri Sants. mayri For. v. chimporensis n. var.

)

))

) st. jucundus Sants.

Polyrhachis (Myrma) wellmani For. schistacea Mayr. cubangensis For. st. sankisianus For.

))

))

))
))

))

))
)

)) v. rugulosa Mayr. atrociliata Sants. v. benguelensis

Soit 139 formes différentes réparties en 93 espèces, 23 races et 22 variétés. Ainsi, M. le $\mathrm{D}^{\mathrm{r}}$ Mon ARD a récolté plus de la moitié des formes connues de l'Angola. Il est curieux de constater la pauvreté des Dolichoderines dans cette liste. Aucune espèce de cette sousfamille n'a été récoltée par M. MonaRd, ni d'ailleurs, par la mission de Rohan-Сhавот. Pourquoi ?

\section{LISTE DES LOCALITÉS DE L'ANGOLA DANS} LESQUELLES ONT ÉTÉ RÉCOLTÉES LES FOURMIS PAR LE Dr MONARD.

Vila da Ponte: sur le Rio Kubango, $280 \mathrm{~km}$. au sud de Huambo. Décembre-janvier 1928-1929.

Rio Tumbolé: affluent du Kutato, lui-même affluent du Kubango. Environ $40 \mathrm{~km}$. à l'est de Vila da Ponte. Janvier 1929.

Rio Mbalé: affluent du Kubango, à $120 \mathrm{~km}$. au sud de Vila da Ponte. Septembre 1928.

Cakindo (Caquindo): sur le Kubango, à 60-70 km. au sud du Rio Mbalé.

Chimporo: à $50 \mathrm{~km}$. à l'ouest de Caimdo ou poste A du Kubango. Novembre 1928. 
Tchitunda: à 35 km. à l'est de Vila da Ponte. 9 janvier 1929.

Planalto. Lobido, III, 1929.

\section{LISTES ET DESCRIPTIONS DES ESPÈCES RÉCOLTÉES PAR LE Dr MONARD.}

Ire Sous-famille Ponerinae Lepel.

Centromyrmex constanciae Arnold.

1915. Ann. of South Afric. Mus., XIV, p. 38, pl. II, fig. 14. ㅎ, 우. Cakindo, 1 ఛ.

Connue de Bulawayo, Sud Rhodésia.

Platythyrea crucheti Santschi.

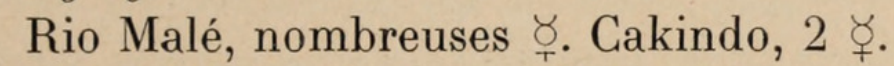

J'ai décrit le type sur trois individus de Cucala (Angola), reçus de J. CRuchet.

Plectroctena mandibularis Sm.

Cakindo, 1 ఛे.

Paltothyreus tarsatus F. v. medianus Sants.

Cakindo, ఫ̛; Rio Mbalé, ఫ̛, (Monard); Cucala (Cruchet).

Outre les caractères distinctifs déjà signalés, cette variété diffère encore du type par la présence d'une petite aire luisante allongée, située devant l'emplacement virtuel de l'ocelle médian et qui manque chez tarsatus Sm.

Megaloponera foetens $\mathrm{F}$. v. crassicornis Gerst.

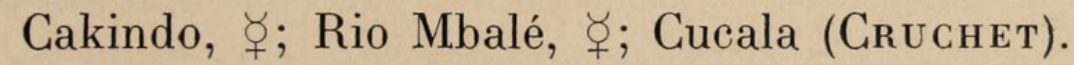

Je rétablis cette variété, confondue avec le type, sur les caractères indiqués dans la clé analytique suivante:

-1. Tibias fortement pileux sur toutes ses faces . . . . . . . . sp. foetens F.

- Pilosité des tibias disposée seulement comme une frange sur le bord fléchisseur.

2. Dos du corps assez abondamment pileux. . . . . . . . . . v. crassicornis Gerst.

- Dos du corps glabre ou à peine pileux . 3

3. Ecaille des grandes et moyennes ouvrières haute, conique, arrondie au sommet. Massif du Kilimandjaro . 
- Ecaille des grandes et moyennes ouvrières plus basse, obliquement tronquée, à profil un peu concave derrière le sommet, plus épaisse chez la petite ouvrière. Bas Congo.

v. termitivora n. var.

La $M$. foetens $\mathrm{F}$. type est répandue au nord du $10^{\circ} \mathrm{L}$. S. jusqu'au Soudan et l'Abyssinie. J'en possède des exemplaires de Brazzaville (A. Werss); Congo Belge: Kassai, Kondué, Luja; Ubanghi (R. P.

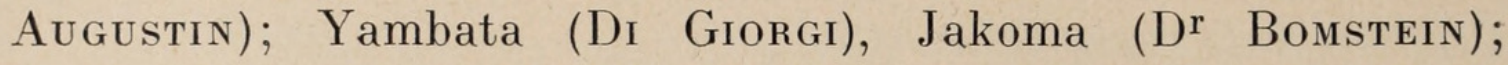
Cameroun: Molundu (Reichensperger); Uganda occidental, Unioro (Alluaud); Afrique orientale anglaise: Pori de Saringhéti (Alluaud); Nyanza (Dr Gérard); Soudan français: Koulouba (Andrieu); Soudan égyptien, Haut Nil Bleu, Roseires (Alluaud); Somalie (FoLEY).

La variété crassicornis Gerst. est de Mozambique, Rhodésia et Angola.

La variété rapax Sants. est de Neu-Moschi et Moschi, dans le massif du Kilimandjaro.

La variété termitivora Sants. de Banana (types) et Moyamba, Bas Congo.

Bothroponera soror Em.

Rio Tumbolé, $\Varangle$; Rio Mbolé, $\Varangle$; Cakindo, $\Varangle$.

Se trouve aussi au Cameroun (type), Togo, Congo belge et français et Rhodésia.

Odontomachus haematodes L.

Chimporo, $\not$, espèce tropicopolite.

II Sous-famille Dorylinae Leach.

Dorylus (Anomma) nigricans Ill. st. molestus Gerst.

Cakindo, $24>\nsucceq$. Race de l'Afrique orientale depuis l'Abyssinie à la Rhodésia du Sud, puis passant à l'ouest par l'Angola. Ceux de cette dernière région ont en général les dents apicales moins recourbées, la basale moins développée. Ceux d'Abyssinie ont la face occipitale plus mate que les exemplaires de l'Afrique orientale anglaise et le Tanganika.

Dorylus (Dorylus) moestus Em. v. claripennis Sants.

Kakindo, $2 \underset{\gamma}{\Varangle}$. (Se trouve aussi dans les provinces voisines du Kassai et du Kwango au Congo Belge.) 
III Sous-famille Pseudomyrminae Emery.

Tetraponera ophthalmica Em. st. angolensis n. st. (fig. 1).

ఛ. Long: $4^{\mathrm{mm}}, 5$. Jaune roussâtre; moitié distale des funicules, une bande transversale sur le troisième tergite du gastre et parfois sur le bord antérieur des deuxième et quatrième tergites d'un brun plus ou moins foncé. Mandibules jaunâtres. Mate ou submate, finement réticulée, avec de courtes rides le long de la suture mésométanotale. Devant des nœuds et du gastre plus finement sculptés et assez luisants. Pilosité dressée assez longue, fine, espacée sur le corps, les scapes et les hanches, manquant sur les tibias. Pubescence fine, clairsemée sur la tête et les appendices, plus dense sur le gastre.

Tête rectangulaire, rétrécie dans sa moitié antérieure, un peu plus que de moitié plus longue que large, avec les yeux aussi grands que chez ophtalmica, mais plus espacés. Tandis que leur intervalle est d'un tiers de la largeur

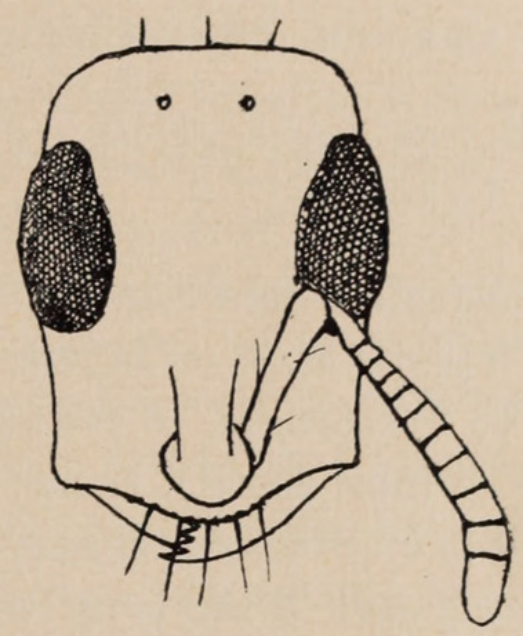

FIG. 1.

Tetraponera ophthalmica Em. st. angolensis $\mathrm{n}$. st. $\not$.

Tête. de la tête chez ophtalmica, il est comme la moitié de cette largeur chez angolensis. Il y a, en outre, deux ocelles latéraux qui manquent chez ophthalmica. L'épistome est arqué devant, avec une crénelure mousse assez faible. Sillon frontal dépassant peu le milieu de la longueur des arêtes frontales. Mandibules de 4 dents. Premier article du funicule plus long que les deux suivants réunis. Thorax comme chez ophthalmica, mais plus robuste, l'épinotum un peu plus anguleux bien qu'arrondi. Stigmates mésonotaux saillants. Le dessous du pédicule est armé d'une dent plus ou moins développée. Du reste comme chez ophthalmica.

Chimporo, $5 \underset{+}{\not}$ (types); Rio Mbalé, $1 \not{q}$.

\section{IVe Sous-famille Myrmicinae Lep.}

Myrmicaria natalensis Sm.

Rio Mbalé, $1 \not{q}$; Tschisenda, $\Varangle$; Cjimporo, $1 \not{q}$. Myrmicaria natalensis Sm. v. nigriventris $\mathrm{n}$. var.

ఛ. Long: $6 \mathrm{~mm}$ à $7 \mathrm{~mm}$. Antennes, pattes, nœuds du pédoncule et 
gastre noirs ou brun noir. Thorax brun rougeâtre, plus clair que la tête. Sauf la médiane, les rides frontales (entre les crêtes frontales) sont effacées, elles se voient avec quelques réticulations dans le lit du scape. Nœuds pédonculaires et gastre entièrement lisses et très luisants. Nœud du pétiole aussi étroit que celui du postpétiole. Pas de crête transversale entre les deux faces du mésonotum. Du reste comme chez natalensis, y compris la forme du pétiole.

Cakindo, $20 \Varangle$; Chimporo, $3 \not{q}$.

Myrmicaria opaciventris Em. v. crucheti Sants.

Cakindo, $\not{\succ}$.

Pheidole rohani Sants. (fig. $2 a, b$ ).

(1925, Mission Rohan-Chabot en Angola et Rhodésia (1912-1925), IV, Formicides, p. 161. ఫ. Fig. 11.)

4 (non décrit). Long: $7 \mathrm{~mm}$ à 9mm. D'un brun rouge foncé, passant au brun noir. Antennes, articulations des pattes et souvent la base du gastre d'un brun de poix plus ou moins clair. Mandibules,

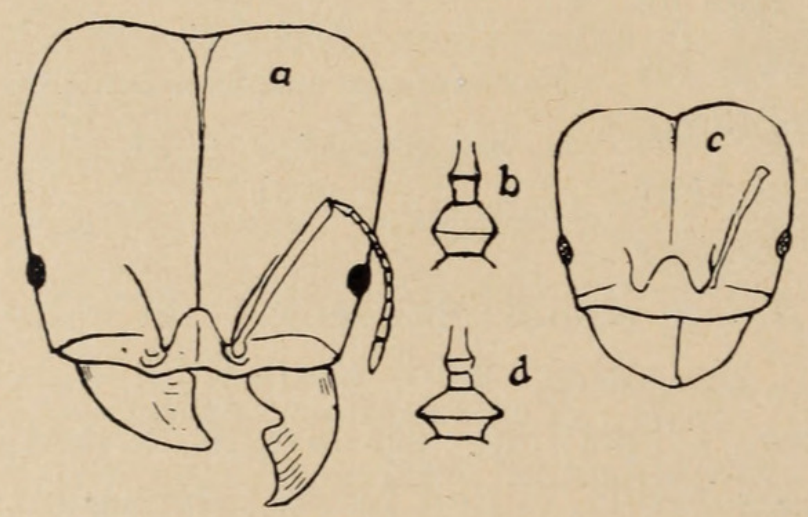

FIG. 2 .

Pheidole rohani Sants.

$a=$ Tête du $\psi ; b=$ Pédoncule vu de dessus.

$P h$. rohani st. pellax

$c=$ Tête du $\nvdash ; d=$ Pédoncule vu de dessus. pédoncule, reste des fémurs, tibias et gastre d'un brun noir ou noirs. Tarses rous sâtre clair. Tête mate, grâce à un fond finement réticulé sur lequel s'étend un réseau de rides longitudinales dans le tiers antérieur, puis de plus en plus divergentes dans les tiers restants. Ces rides assez fines sont réunies par des trabécules qui abondent surtout vers les lobes occipitaux. Cette sculpture ressemble à celle de $P h$. excellens Mayr, mais estplus

fine. Thorax réticulé ponctué comme la tête, mais plus faiblement sur le pronotum et le mésonotum où se trouvent des rides transversales irrégulières. Des rides grossières, irrégulières et longitudinales sur les côtés du thorax. Nœuds du pédoncule réticulés, avec quelques rides transversales. Gastre et appendices lisses et luisants. Pilosité dressée abondante sur le thorax et le gastre, 
moins sur la tête; les pattes n'ont qu'une forte pubescence oblique.

Tête carrée (longueur du bord antérieur au sommet du lobe occipital: chez le soldat de $9 \mathrm{~mm}, 2^{\mathrm{mm}}, 9$ sur $3^{\mathrm{mm}}$ de large et chez celui de $7 \mathrm{~mm}, 2^{\mathrm{mm}}, 5$ sur $\left.2^{\mathrm{mm}}, 5\right)$. Les côtés moyennement convexes, largement échancrés en triangle derrière comme chez $P$ h. excellens. Sillon frontal continu avec la gouttière occipitale complète. Epistome caréné, faiblement mais assez largement échancré devant. Mandibules lisses et luisantes dessus avec quelques stries vers la base de la face externe; le bord terminal tranchant, mousse au bout ou faiblement tuberculé. Le scape atteint le milieu de la tête. Articles 3 à 5 du funicule un peu plus longs qu'épais, les autres plus longs. Le dernier de la massue aussi long que les deux précédents réunis. $\mathrm{Vu}$ de dessus le pronotum est plus arrondi dans ses côtés que chez excellens. Mésonotum avec un bourrelet transversal peu saillant au milieu, plus accusé aux bords, qui paraissent dentés chez certains individus. Face basale de l'épinotum aussi large que longue, avec les bords divergents vers les épines. Celles-ci, verticales, moins longues et moins divergentes que chez excellens, longues comme la moitié de l'intervalle de leur base. Sommet du pétiole échancré. Postpétiole trois fois plus large que le pétiole, les côtés prolongés en épines un peu recourbées en arrière comme chez excellens, mais plus fortes. Gastre non échancré à la base. Cuisses un peu plus épaisses, que chez excellens $\not$. Comme je l'ai dit, cette ouvrière a sur les côtés du pronotum un peu avant de la suture mésonotale, un tubercule dentiforme surmonté d'un poil. Cette disposition se retrouve chez d'autres espèces, par exemple chez $P h$. rhodesiana ${ }^{1}$ et liengemei For. Les ouvrières récoltées par M. Monard sont en général plus obscures que celles sur lesquelles j'ai établi cette espèce.

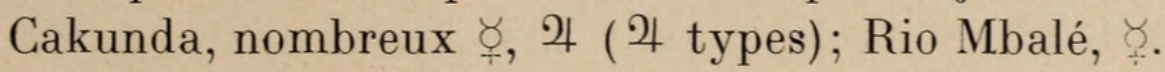

Voisine aussi de Ph. saxicola Wheel., mais plus sombre, le postpétiole plus large tant chez le soldat que chez l'ouvrière, et les antennes plus longues.

Pheidole rohani Sants. st. pellax n. st. (fig. $2, c, d$ ).

2. Long.: $5^{\mathrm{mm}}, 2$. Noir. Mandibules, antennes, articulations des

1 Pheidole sculpturata Mayr st. rhodesiana (Forel), syn. Ph. excellens st. rhodesiana Forel 1913. - FoREL avait rapporté avec doute cette race à excellens; en effet, la tête allongée du soldat et sa sculpture la placent tout près de sculpturata. 
pattes et tarses brunâtres. Pilosité dressée assez abondante, longue sur le corps, plus courte sur les fattes, réduite à une pubescence oblique sur les scapes. Pas de pubescence adjacente sur le corps. Tête assez régulièrement et fortement ridée en long dans sa moitiéantérieure et en divergeant dans l'autre moitié. Ces rides deviennent réticulaires vers les lobes occipitaux. Leurs intervalles sont plus ou moins finement réticulés rugueux, mats ou submats. Les rides sont plus fines et plus serrées entre les arêtes frontales. Thorax réticulé ponctué, avec des rides transversales sur le pronotum, longitudinales sur le mésonotum et convergentes vers les épines de l'épinotum. Pédoncule faiblement réticulé, avec quelques rides fines, transversales. Gastre et pattes lisses.

Tête presque carrée, longue de $2^{\mathrm{mm}}, 1$, large de $2^{\mathrm{mm}}$. Les côtés faiblement convexes, le bord postérieur largement mais peu profondément concave. Le sillon frontal continue avec le sillon occipital. Yeux ovales entre le quart et le tiers antérieur des côtés. Arêtes frontales plus espacées que longues. Aire frontale lisse, fortement imprimée derrière. Epistome faiblement convexe derrière, plutôt plat devant, avec une forte ride médiane espacée, d'autres rides plus faibles. Le bord antérieur faiblement échancré. Mandibules subdentées au bout, lisses, avec de gros points espacés, mais striées à leur base. Le scape récliné vers l'angle postérieur de la tête atteint le tiers postérieur de celle-ci. Deuxième article du funicule aussi large que long, les suivants de plus en plus longs. Dernier article du funicule moins long que l'ensemble des deux précédents qui sont subégaux. Pronotum plus long que large, à côté anguleux et suture effacée. Mésonotum faiblement convexe, au milieu un bourrelet peu marqué. La face basale de l'épinotum bordée seulement vers les épines et presque aussi large que longue jusqu'à la base des épines. Celles-ci sont larges, épaisses à la base, assez fines pour le reste, assez relevées et peu divergentes, longues comme la moitié environ de la face basale. La face déclive presque droite. Le nœud du pétiole squamiforme, échancré au sommet. Postpétiole deux fois plus large que le pétiole et que long; les côtés anguleux, subdentés, le dessous arrondi sans saillie. Gastre court, non tronqué à la base.

ఛ. Long.: $3^{\mathrm{mm}}$ environ, noire, appendices bruns. Ne diffère de rohani que par la tête un peu plus étroite.

Rio Mbalé, 나, ㅃ․ 
Pheidole excellens Mayr, st. weissi Sants. v. mbalensis n. var. (fig. 3, $a, b, c, d)$.

ఛ. Diffère du type ${ }^{1}$ par sa couleur noire, les appendices bruns foncés, les cuisses presque noires. Les articulations des pattes brun jaunâtre. Pas de rides surajoutées sur le thorax qui est réticulé-ponctué comme la tête et la base du gastre. Les yeux un peu plus allongés et relativement plus grands.

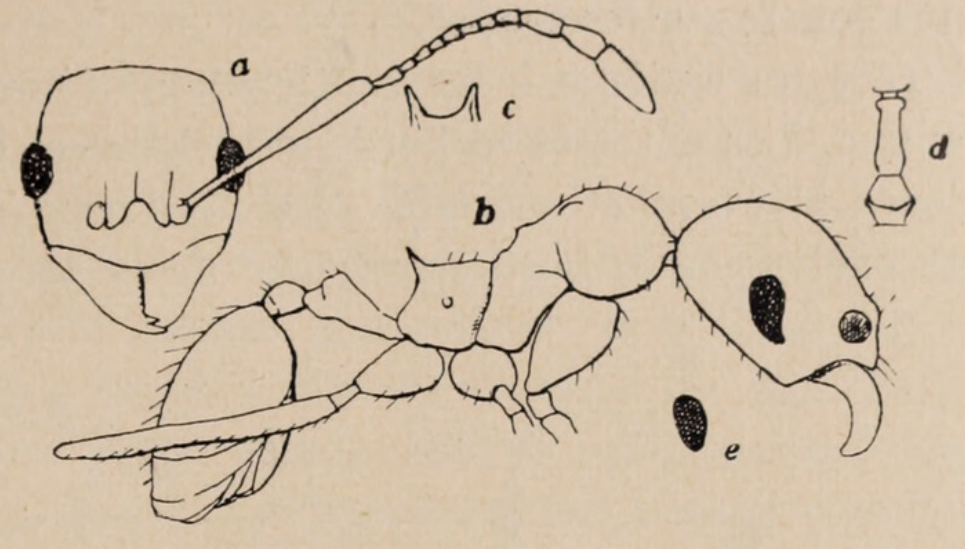

FIG. 3.

Pheidole excellens Mayr st. weissi Sants. v. mbalensis $\mathrm{n}$. var.

Les tubercules du pronotum un peu plus saillants. Pour le reste comme chez weissi Sants.

Rio Mbalé, 15 ఛ; Timbolé, 1 \%.

Pheidole monardi n. sp. (fig. 4, $a, b, c)$.

ఛ. Long.: $3^{\mathrm{mm}}, 7$. Noire, appendices d'un brun moyen avec les tarses brun jaunâtre. Tête, moins le front, thorax et pédoncule, moins le dessus des nœuds, régulièrement réticulés, ponctués et mats, sauf sur le vertex, où la sculpture s'efface un peu et paraît luisante. Quelques fines rides espacées partent du devant des joues, contournent les yeux et atteignent les angles postérieurs de la tête. Quelques rides plus accusées transversales sur le devant du

1 Pheidole excellens Mayr st. weissi Sants. (fig. 3 e).

$\Varangle$ (non décrite). Long.: $3 \mathrm{~mm}$. Brun roussâtre foncé, gastre plus roussâtre, appendices jaunâtres. Partout régulièrement réticulée ponctuée en dé à coudre sauf sur la moitié postérieure du premier tergite du gastre, les segments suivants et les pattes. Mandibules striées. Quelques faibles rides sur les joues et le promésonotum. Pilosité dressée assez longue et abondante, plus courte et oblique sur les pattes.

Tête rectangulaire, un peu plus large (avec les yeux) que longue. Son bord postérieur faiblement arqué, masquant le bord cervical lequel est court et faiblement bordé. Angles postérieurs de la tête brèvement convexes, les côtés assez convexes. Vu de face les yeux paraissent à peine en avant du milieu des côtés, mais vus de côté, ils se prolongent en se rétrécissant en avant et en dessous (fig. $3 e$ ), leur grand diamètre étant un peu plus court que leur distance au bord antérieur de la tête. Sillon frontal court, assez large, atteignant le milieu de la tête. Arêtes frontales parallèles, aussi espacées que longues. 
pronotum et obliques sur les côtés de l'épinotum, le reste lisse et luisant. Une longue et fine pilosité sur le corps et les appendices, plus courte sur les scapes.

Tête un sixième à un septième plus large, avec les yeux, que longue. Les côtés et le bord postérieur convexes, les angles postérieurs brièvement arrondis. Le bord cervical bordé, assez large, est caché par le bord postérieur de la tête dans la vue frontale.

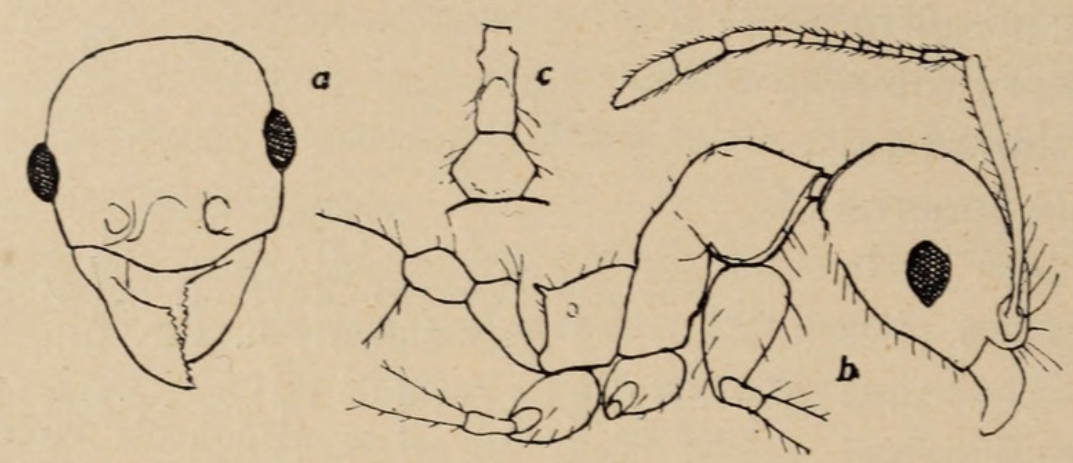

FIG. 4.

Pheidole monardi n. sp. $\not$.

$a=$ Tête; $b=$ Profil de l' $\not$; $c=$ Pédoncule vu de dessus.

Les yeux assez convexes sont grands comme le quart des côtés de la tête. Sillon frontal court, large devant. Arêtes frontales parallèles devant, à peine divergentes derrière. Aire frontale et milieu de l'épistome lisses. Celui-ci caréné, à bord antérieur arqué, sans échancrure. Mandibules striées, armées de 10 à 12 dents, les apicales plus fortes. Le scape dépasse d'un quart le bord postérieur de la tête. Articles deux à huit de $1 \frac{1 / 2}{2}$ à 2 fois plus longs qu'épais.

Aire frontale lisse. Epistome convexe, arqué devant avec une ride médiane plus forte que les latérales qui sont assez espacées. Mandibules de 10 à 11 dents, les apicales plus fortes. Le scape dépasse d'un quart de sa longueur le bord postérieur de la tête. Articles moyens du funicule plus longs qu'épais, le dernier pas beaucoup plus long que chacun des deux précédents. Pronotum assez convexe, aussi large que long avec le cou, les angles postérieurs faiblement tuberculés. Bourrelet du mésonotum peu saillant sur le profil. Face basale de l'épinotum plane, près de deux fois plus longue que large au milieu. Epines assez relevées, longues comme le tiers de cette face. La face déclive un peu plus courte que la basale, du reste semblable. Nœud du pétiole triangulaire, peu ou pas échancré au sommet. Postpétiole deux fois plus large que le pétiole, aussi large que long, ses côtés anguleux.

Congo français: Brazzaville $\not$ (types), M'Douna, $\Varangle$ (A. Weıss). J'ai décrit le soldat en 1907 , les ouvrières étaient dans le même tube mais séparées du soldat. L'identité n'est donc pas absolue. Cette forme est plus petite et moins ridée que chez excellens Mayr, les tubercules du pronotum moins accusés. 
Dernier article de la massue plus court que les deux précédents réunis, ces derniers deux fois et demi plus longs qu'épais. Pronotum aussi long que large avec le col, les côtés très convexes, les angles postérieurs légèrement tuberculés. La suture promésonotale peu distincte sur le dos. Mésonotum long, faiblement convexe sur le profil. Face basale de l'épinotum deux fois plus longue que large, un quart plus large derrière, les côtés bordés, plane ou faiblement concave. Epines un peu plus longues que le quart de la longueur de la face basale, bien plus courtes que l'intervalle de leur base, obliques en dehors et en arrière. Face déclive presque verticale, assez plane, non bordée. Nœud du pétiole triangulaire, à sommet brèvement tronqué, sans échancrure. Postpétiole deux fois plus large que le pétiole, légèrement plus large que long, les côtés anguleux à sommet au tiers postérieur. Premier tergite du gastre un peu plus long que large derrière.

Rio Mbalé, 10 ૪̆.

Le soldat n'a pas été capturé. Il paraît devoir se rapprocher du groupe excellens sculpturata au postpétiole fortement transversal et denté.

Pheidole victoris For.

Chimporo, 2 ㄴ, 2 ఛ.

Cette forme est très voisine de $P$ h. tenuinodis Mayr. La comparaison de deux types ne montre que des différences minimes. Peut-être une simple race ou variété.

Pheidole megacephala F.

Rio Mbalé, 24, ఫ̛; Tchituada, ㅇ, ఫ̛.

Pheidole megacephala F. v. atrox For.

Rio Mbalé, 나, 후, 우.

Pheidole megacephala F. v. nkomoana For.

Rio Mbalé, ఛ̧, ঋ.

Pheidole megacephala F. st. punctulata Mayr. ? v. talpa Gerst.

Rio Mbalé, ఫ̛, 나, ㅇ.

Variété foncée. La punctulata Mayr. type est claire, j'en possède

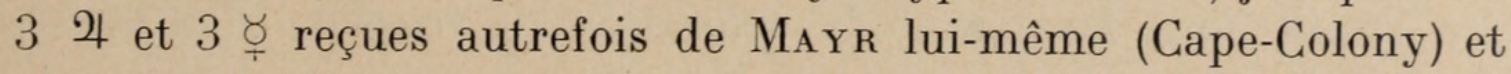
correspondant exactement à la description initiale de 1866. Quand à la forme foncée, elle serait peut-être bien la v. talpa Gerst. Elle 
est plus grande que atrox For. et la sculpture ponctuée et réticulée plus accusée sur les lobes occipitaux.

Pheidole sp.?

Chimporo, 4 ; ; Cakindo, ㅇ.

Indéterminables sans les $\mathcal{\vartheta}$ et $\breve{q}$.

Ocymyrmex weitzekeri Em. st. monardi n. st.

ఛ. Long.: $7 \mathrm{~mm}$ à $8 \mathrm{~mm}$. D'un brun rouge foncé. Abdomen et cuisses noirs. Antennes, tibias et tarses un peu plus clairs que le thorax (parfois la base du gastre est brunâtre et le reste de l'insecte plus clair). Le front et les côtés de la tête en dehors des yeux sont ridés striés en long et le fond plus ou moins ponctué réticulé. Les stries frontales n'atteignent pas le bord postérieur de la tête, loin de là, mais elles deviennent serpigineuses ou réticulaires dès le vertex. Tout le reste de la tête est réticulé ponctué avec quelques rides irrégulières. Pronotum ridé strié en long. Reste du thorax strié ridé en travers. Abdomen lisse et luisant. Tête un peu moins élargie devant que chez weitzekeri, plutôt comme chez la race wroughtoni For. Les articles du funicule plus longs que chez ce dernier. Echancrure médiane du bord de l'épistome un peu plus faible. La face déclive de l'épinotum descend obliquement. Elle est moins abrupte que chez wroughtoni et surtout que chez transversus Sants. chez qui elle est presque verticale. Pétiole étroit et allongé comme chez fortior For. et arnoldi For., son nœud plus long que large. Le postpétiole piriforme, deux fois plus long que iarge derrière. Gastre mince à la base.

Race distincte de toutes celles du weitzekeri par la sculpture de la tête.

Cakindo, $7 \Varangle$ (types); Chimporo, $12 \not{q}$. Quelques exemplaires plus clairs sont d'ailleurs semblables, avec la même sculpture caractéristique de la tête.

Ocymyrmex picardi Forel.

Cakindo, $\breve{q}$; Chimporo, $\breve{q}$.

Crematogaster (Acrocoelia) castanea Sm. v. bruta Sants.

Chimporo, $\Varangle$. Cette variété est presque aussi ridée sur le thorax que simia For., mais avec la coloration de tricolor, plus claire chez simia.

Chimporo, $\not$. 
Crematogaster (Acrocoelia) castanea Sm. v. arborea Sm.

[Syn. $C r$. (Ac.) castanea Sm. v. decolorata Santschi 1925. Rev. Zool. Afr. XIII, p. 213.]

Crematogaster (Acrocoelia) castanea Sm. st. inversa For. v. flaviventris Sants.

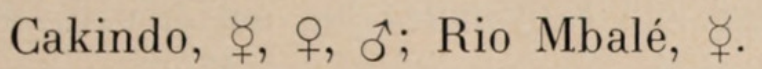

Crematogaster (Acrocoelia) castanea Sm. st. inversa v. mossamedana

n. var.

$\Varangle$. Diffère du type par sa couleur d'un brun rouge foncé; les appendices, surtout les antennes sont presque noirs. Gastre jaune ocré avec le bout plus ou moins brunâtre. Du reste comme chez la var. flaviventris, mais celle-ci a le corps et les appendices d'un rouge ferrugineux.

ㅇ. Long.: $8^{\mathrm{mm}}$. Noire, le gastre jaune ocré un peu rembruni au bout, parfois le bord postérieur des derniers segments nuagé d'un brunâtre plus clair que chez flaviventris.

Mbalé, $\not$, $q$ (types); Chimporo, $\Varangle ;$ Tchitunda, 1 q; Timbolé, $\Varangle$ plus petites.

Crematogaster (Acrocoelia) gerstaeckeri D. T. st. bulawayensis For.

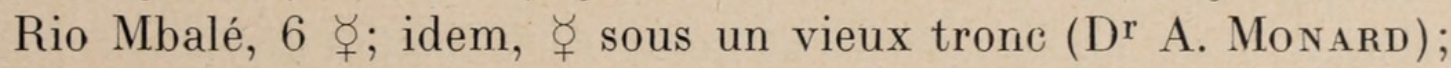

Cakindo, $\breve{q}$.

Le $C r$. gerstaeckeri D. T. (-Cr. cephalotes Gerst.) est, selon des exemplaires reçus déterminés par M. Menozzi très voisin de la race pulla Sants. Il découle de cela que les races et variétés se rattachant à cette espèce doivent être disposées comme suit:
Cr. gerstaeckeri D. T.
v. pulla Sants.
st. godefroyi For.
v. pudica Sants.
st. oraclum For.
st. bulawayensis For.
st. sjöstedti Mayr
v. inquieta Sants.
v. infaceta Sants.
v. zulu Sants.
v. rufescens Sants.
v. kohliella For.

Crematogaster (Sphaerocrema) amita For.

Chimporo, $\not$.

Crematogaster (Atopogyne) clariventris Mayr st. biimpressa Mayr.

Tumbolé, 1 ఛ.

Solenopsis punctaticeps Mayr.

Chimporo, $1 \not{q}$.

Rev. Suisse de Zool. T. 37. 1930. 
Monomorium (Xeromyrmex) salomonis L. st. junodi For. v. opacior For.

Rio Mbolé, 1 ఛ.

Monomorium (Monomorium) setuliferum For.

Chimporo, 1 petite $\not{q}$.

Emery a basé principalement sur la forme des articles de la massue antennaire sa distinction entre les sous-genres Xeromyrmex et Monomorium. Or, le petit groupe que cet auteur a rangé autour $M$. pharaonis se place exactement entre ces deux sous-genres, il a la massue comme chez Monomorium et la sculpture mate des Xeromyrmex. A ce groupe il faut ajouter M. hannonis Sants.

Cardiocondyla (Loncyda) n. sg. ${ }^{1}$.

Diffère du sous-genre Cardiocondyla par le thorax inerme, sans suture ni impression dorsale. Le pétiole très allongé, le post-pétiole plus long que large. La pubescence squameuse. Pas de poils dressés.

Cardiocondyla (Loncyda) monardi n. sp. (fig. 5, a, b. c).

ఛ. Long.: $2^{\mathrm{mm}}, 6$. Roux jaunâtre, appendices plus clairs, le dessus du gastre plus ou moins rembruni. Mate, très finement réticulée, avec une pubescence squameuse, large à la base, pointue au bout, d'un blanc argenté et dense sur tout le corps.

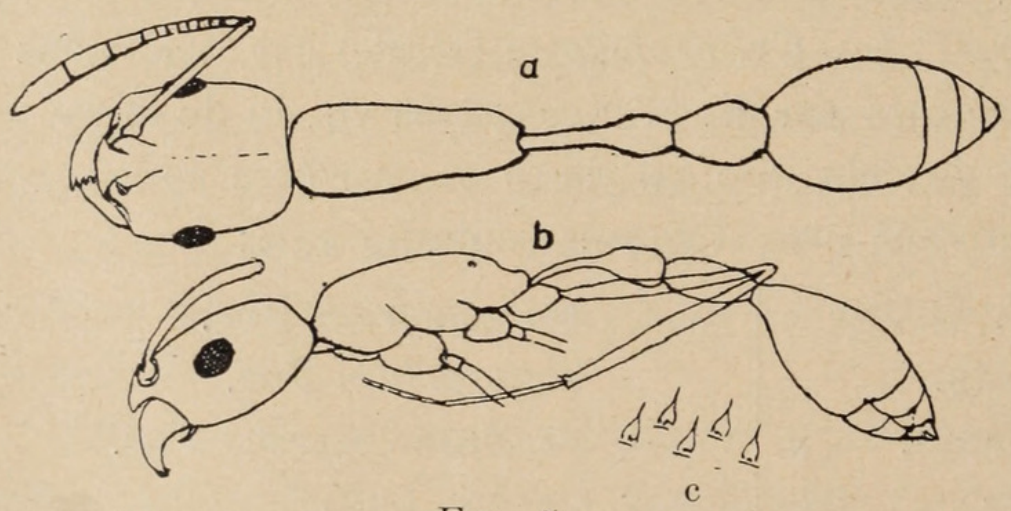

Fig. 5.

Cardiocondyla (Loncyda) monardi n. sg. n. sp. ఫ̆. $a=\mathrm{Vu}$ de dessus $; b=\mathrm{Vu}$ de profil $; c=$ Pilosité fortement grossie.

Tête rectangulaire, un quart plus longue que large, les côtés faiblement convexes, le bord postérieur droit, avec les angles arrondis. Les yeux sont placés en avant du milieu des côtés dont ils représentent le quart environ. Aire frontale courte.

1 J'ai décrit en 1912 sous le nom de Monomorium cristatum une Cardiocondyla aberrante de Madagascar. Cette espèce diffère cependant des autres espèces du genre par la pilosité dressée assez abondante de l'abdomen et l'étranglement accentué du thorax. Elle mérite pour cela d'entrer dans le cadre d'un nouveau sous-genre que je nomme Dyclona n. sg., nom qui, comme celui de Loncyda est un anagramme de Condyla. Ce nouveau sous-genre fait passage entre les Cardiocondyla et les Monomorium. 
Un très léger sillon frontal atteint le vertex. Aire frontale indistincte. Bord antérieur de l'épistome arqué, le bord postérieur échancré en bourrelet devant les fosses antennaires. Le scape atteint le bord postérieur de la tête. Articles 3 à 6 du funicule aussi longs qu'épais, les autres de plus en plus longs. Les deux premiers articles de la massue un peu plus de deux fois plus longs que larges. Le dernier encore plus long et plus épais. Thorax deux et demi fois plus long que large, non bordé, sans suture sur le dos. L'épinotum inerme, sa face déclive concave. Pétiole très longuement pédiculé devant, arqué, avec le nœud bas, deux fois plus long que large et aussi long que les deux tiers de son pédicule. Postpétiole déprimé, légèrement plus haut et deux fois plus large que le pétiole, un bon tiers plus long que large au tiers postérieur où il est le plus ample. Gastre court comme le thorax. Pattes très allongées.

Rio Mbalé, $2 \Varangle$ (types); Chimpora, $1 \Varangle$, variété plus obscure, la tête, massue de l'antenne, dessus des nœuds et gastre brunâtres.

Tetramorium setuliferum Em. v. cucalense Sants.

Chimporo, $\not$,, .

La var. triptolemus Arn. me parait synonyme.

Tetramorium quadrispinosum Em. st. angolense n. st.

ఛ. Long.: $3 \mathrm{~mm}, 2$. Rouge roussâtre. Gastre noir avec la base brunâtre. Appendices jaune roussâtre. Tête mate, à peine plus fortement ponctuée-réticulée que chez le type, sans fossette, mais avec des rides frontales atteignant en partie le vertex et le bord postérieur de la tête. Les angles postérieurs assez lisses. Les joues grossièrement ridées-réticulées. Thorax assez fortement ridé, en long sur le pronotum, en travers sur le mésonotum et l'épinotum. Pas de rides sur les côtés du thorax qui sont régulièrement, mais plus fortement réticulés que la tête. Les deux nœuds grossièrement ridés-réticulés sur le même fond ponctué que la tête. Gastre très finement strié en long et mat dans la moitié basale du premier tergite, le reste lisse et luisant. Quelques longs poils vers la bouche, les hanches et sous le gastre, absents ailleurs. Pubescence très courte et rare.

Tête comme chez quadrispinosum Em., à peine plus étroite; l'épistome avec la même carène. Thorax un peu moins convexe. Les nœuds un peu plus étroits. Le postpétiole aussi long que large derrière, les côtés convergeant en avant et plus rectilignes. Pour le reste comme chez le type dont il diffère surtout par la sculpture. 
․ Long.: $5^{\mathrm{mm}}$. Couleur de l'ouvrière. La tête est bien plus fortement sculptée. Tout le dessus du gastre mat. Mésonotum et scutellum ridés en long. Pronotum et épinotum ridés en travers. Pilosité et le reste comme chez l'ouvrière.

Cakindo, 1 ఛ, 1 q. Cette dernière, bien que de la même localité, n'a pas été prise dans le nid avec les ouvrières et son identité reste un peu douteuse.

Atopomyrmex mocquerysi André st.opacus Sants. v. nigellus n. var.

ఛ. Même sculpture mate de tout le gastre et de toute la tête avec la même ponctuation entre les rides de celle-ci que chez opacus, dont cette variété diffère seulement par la couleur noire du gastre des grandes ouvrières, couleur qui s'étend sur presque tout le corps chez les petites. Chez celles-ci seul le devant de la tête reste brun rougeâtre tandis que chez les grandes et moyennes toute la tête est rouge foncé. Le thorax des grandes $\Varangle$ est d'un rouge plus foncé que la tête. (Chez opacus la tête et le thorax sont d'un rouge clait et le gastre brun roussâtre.)

Rio Mbalé, $\not<\Varangle ;$; Chimporo, 1 q.

Cataulacus baumi For.

Mbalé, 2 q. Légère variété à fémurs rouge foncé.

VIe Sous-famille Formicinae (Lep,) Forel emend.

Anoplolepis (Mesanoplolepis) trimeni For. v. angolensis n. var.

ఫ. Long.: $2^{\mathrm{mm}}, 6$ à $3^{\mathrm{mm}}$ suivant l'état de réplession du gastre. D'un jaune roussâtre plus clair que chez déceptor Arn. Derniers articles du funicule et à peine les côtés du gastre brunâtres. Pour le reste comme chez trimeni For.; mais la tête est plus rectangulaire, moins rétrécie devant.

Rio Mbalé, 3 ఛ .

Dans ma description de ce sous-genre (Ann. Soc. Ent. France $\mathrm{XCV}$, p. 14, 1926) je dis qu'il est monomorphe. Il faut entendre par celà que la tête et le thorax de l'ouvrière ne varie pas autant que dans le sous-genre Zealleyella Arn., mais que le gastre peut atteindre de fortes proportions chez certains individus aptes à emmagasiner des réserves alimentaires dans leur jabot distendu. 
Anoplolepis (Zealleyella) braunsi For.

Chimporo, 2 ๖. Le premier article du funicule est plus long que le troisième, ce qui fait que cette espèce n'appartient pas au s. g. Anoplolepis sens strict, comme je l'ai écrit par erreur dans le travail ci-dessus cité.

Plagiolepis (Anacantholepis) fuscula Em.

Chimporo, 1 ఛ. Le type est du Cap.

Acantholepis rugithorax n. sp. (fig. $6, a, b$ ).

†. Long.: $3 \mathrm{~mm}, 3$. D'un brun rouge foncé presque noir, côtés des mandibules, scapes et premier article du funicule roussâtres. Reste des mandibules, base des cuisses et des tibias, côtés du mésonotum brun rougeâtre. Gastre noir. Seulement quelques poils sur le bord de l'épistome, plus courts et épais au bout du gastre. Pubescence adjacente très fine, courte et clair-semée.

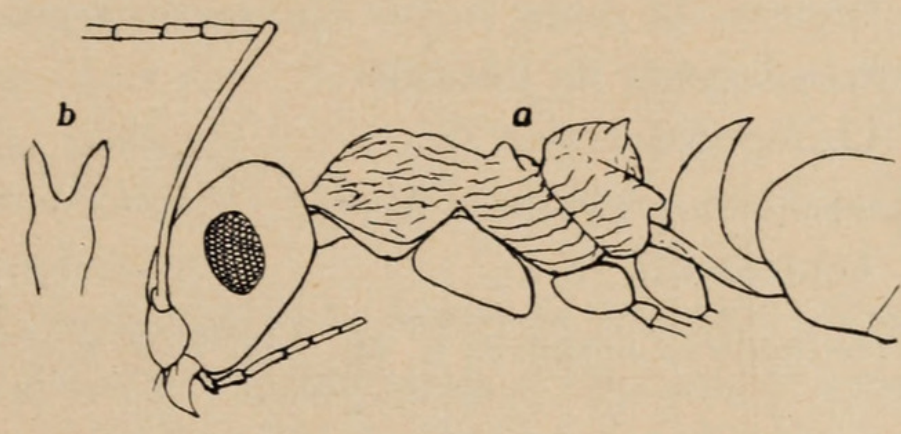

FIG. 6. Acantholepis rugithorax n. sp. $\not$. $a=$ Profil $; b=$ Ecaille vue de face.

Mate. Tête finement et densément réticulée-ponctuée partout ainsi que l'écaille et le gastre, mais les côtés de ce dernier, l'épistome et les pattes sont encore plus finement sculptés. Le thorax, au contraire, est grossièrement rugueux, surtout l'épinotum. Le pronotum et les côtés du thorax sont en outre ridés en long ou en travers.

Tête ovale, un peu plus large que longue, les angles postérieurs fortement arrondis derrière les yeux, le bord postérieur faiblement convexe, les côtés droits et assez convergents en avant; les yeux sont un peu moins grands que la moitié des côtés de la tête. Arêtes frontales parallèles, plus longues que leur intervalle. Sillon frontal très court. Aire frontale large, mate. Epistome caréné, fortement arqué devant. Mandibules finement striées, leur bord terminal oblique avec une forte dent apicale. Le scape dépasse d'un peu plus de sa moitié le bord postérieur de la tête. Deuxième article du funicule près de deux fois plus long que large, les suivants bien deux 
fois plus longs que larges, subégaux en longueur, mais s'épaississant de plus en plus. Pronotum bordé devant, à épaules anguleuses, les bords latéraux droits convergent en arrière où ils s'arrondissent. Mésonctum cylindroconique, un peu plus long que large devant. Métanotum un peu plus large que long. Les stomates élevés, distants de presque le double de leur diamètre. Epinotum très relevé devant, la face basale convexe sur le profil, en rectangle un peu plus large que long, avec une petite épine mousse aux angles postérieurs. Face déclive, convexe de haut en bas, avec un tubercule saillant de chaque côté de l'articulation. Ecaille bien plus haute que son pédicule postérieur. Vue de face elle dessine un rectangle d'un tiers plus haute que large, légèrement convexe au milieu des côtés et surmonté de deux fortes épines divergentes, un peu plus longues que la largeur de l'écaille.

Chimporo, $1 \not{q}$.

Acantholepis capensis Mayr.

Tehituanda, $3 \not{q}$.

Acantholepis monardi $\mathrm{n}$. sp. (fig. $7, a, b$ et $8, b$ ).

b
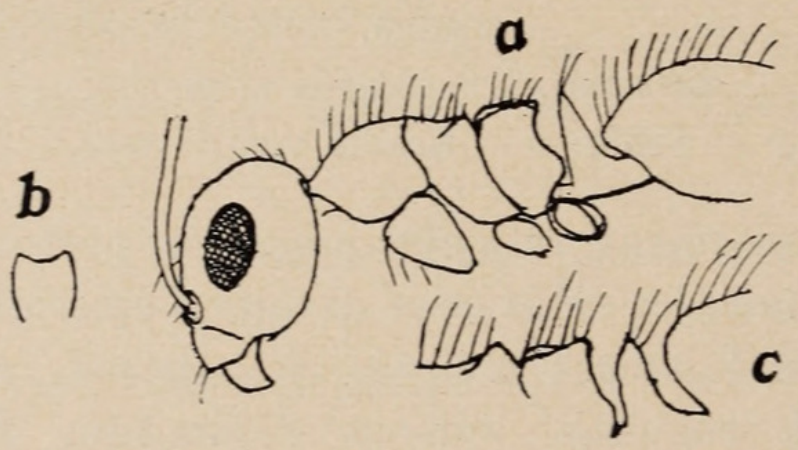

Fig. 7.

Acantholepis monardi n. sp. $\Varangle$.

$a=$ Profil du corps $; b=$ Ecaille vue de

face; $c=$ Var. australis $\mathrm{n}$. var., profil de l'épinotum et de l'écaille. ф. Long.: $2^{\mathrm{mm}}, 2$. Noire. Mandibules, scape et tarses roussâtres. Funnicules brunâtres. Luisante, lisse. Quelques fines rides longitudinales sur les côtés du mésonotum. Pilosité dressée blanchâtre, fine, assez longue et abondante sur le corps, plus rare sur la tête, courte sur les pattes. Une pubescence clairsemée sur la tête, rare ou nulle sur le reste du corps, dense et fine sur les antennes et les pattes.

Tête rectangulaire, un peu plus longue que large, un peu plus étroite que chez capensis Mayr, à peine plus large derrière que longue, les côtés et le bord postérieur faiblement convexes, les angles arrondis et loin des yeux, lesquels sont plus petits que chez capensis. Sillon frontal nul. Epistome caréné. Le scape dépasse d'un quart le bord occipital. Les articles du funicule plus courts que chez 
capensis et canescens. Le quatrième un tiers à une demi fois plus long qu'épais. (Deux fois plus long qu'épais chez canescens.) Thorax plus étroit que la tète et moins allongé que chez canescens. Les côtés du pronotum sont assez fortement et régulièrement convexes, comme chez cette dernière.

Métanotum moitié plus large que long, les éminences à stigmates ne dépassent pas la hauteur de l'épinotum. La face basale de celui-ci est presque horizontale, à peine relevée derrière, très élargie aux angles qui sont tuberculés et échancrés entre eux. La face déclive concave en haut. Pétiole à écaille deux fois environ plus haute que le pédicule postérieur. Le sommet modérément échancré est tranchant sur le profil.

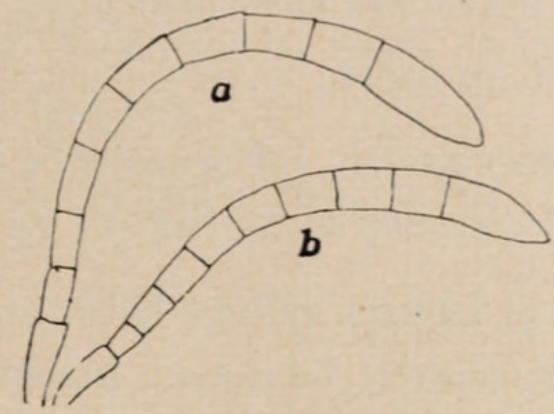

FIG. 8

Funicules de Acantholepis

a) canescens Em.;

b) monardi n. sp.

La base du gastre recouvre le pédicule postérieur du pétiole. Voisine de A. crinata Mayr. Mais celle-ci a le mésonotum et l'épinotum grossièrement sculptés. Chez canescens Em. (fig. 8 a) les articles antennaire. sont plus longs, ainsi que le thorax. Chez capitata For., le scape est plus court que la tète. Chez curta Emery, le corps est plus ramassé, le métanotum plus de deux fois plus large que long.

Timbolé, 2 ఛ.

Acantholepis monardi Sants.v. australis n. var. (fig. 7, c).

ఛ. Long.: $2^{\mathrm{mm}}, 5$. Diffère du type par la pilosité blanche plus longue sur le corps et plus abondante sur la tête. Celle-ci est un peu plus large. Le deuxième article du funicule un peu plus court. Le devant de la face basale de l'épinotum moins relevé et l'éminence du stomate métanotal plus dégagé et relativement plus haut. L'écaille plus épaisse vers le haut, où les faces antérieures et postérieures sont légèrement convexes et le sommet presque aussi tranchant et échancré que chez le type.

Tchitunda, $2 \Varangle$ (types); Chimporo, $1 \not$.

Camponotus (Tanaemyrmex) wellmani For.

Tchitunda, 9, I, 29. " $\not, \not{q} »$.

Camponotus (Myrmoturba) maculatus F. st. atramentarius For. v. cluis For.

Timbolé, Ұ. 
Camponotus (Myrmoturba) maculatus F. st. manzer For.

Chimporo, $\Varangle<\Varangle$. Se trouve aussi en Rhodésia. La var. diffusus Sants. se rapporte à manzer Forel et non à radamoides For.

Camponotus (Myrmoturba) maculatus F. st. radamoides For. v. liocnemis Em. (fig. 9, a).

Chimporo, $\breve{q}$.

Camponotus (Myrmoturba) maculatus F. st. radamoides For. var. calceatus n. v. (fig. 9, b).

ఛ. Long.: $13^{\mathrm{mm}}$. Tête, mandibules, scapes, dos du thorax, genoux antérieurs, tibias et métatarses des deux dernières paires, gastre moins les taches et les bords des segments, noirs ou noirâtres. Le reste jaune ocré. Cuisses et taches du gastre plus claires. Ces dernières sont plus nettement limitées que chez liocnemis Em., elles n'atteignent pas le bord postérieur du segment. Les deux taches du premier tergite ne se touchent que près de l'articulation du pétiole et s'étendent sur tout le sternite. Celles du tergite 3 s'étendent
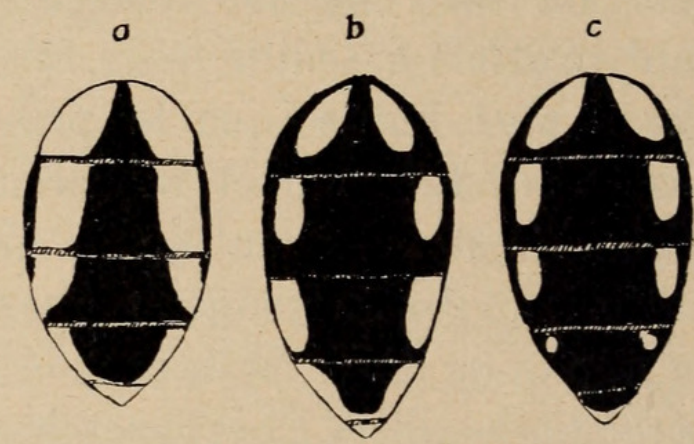

FIG. 9.

Abdomen vu de dessus des " $\not$ de Camponotus (Myrmoturba) maculatus $\mathrm{F}$. $a=$ st. radamoides $\mathrm{v}$. liocnemis $\mathrm{Em}$.; $b=\mathrm{v}$. calceatus n. var.; $c=\mathrm{v}$. citinus n. var. plus en arrière et celles du 4 se touchent même derrière. Tête plus mate que chez liocnemis et plus allongée (longue de $3^{\mathrm{mm}}, 5$, large de $3 \mathrm{~mm}$ ). Tibias postérieurs longs de $3 \mathrm{~mm}, 8$, le bord postérieur sérié de petits poils raides mais moins couchés que chez liocnemis et bien plus petits que les piquants du maculatus. Gastre assez luisant. Ecaille un peu plus épaisse.

ఛ. Long.: $8 \mathrm{~mm}$. Mandibules, milieu du funicule, genoux, métatarses et trois bandes longitudinales sur le gastre d'un brun plus ou moins foncé. Reste des scapes, dos du thorax, tibias postérieurs d'un brun plus ou moins roussâtre. Reste des pattes jaune clair et reste du corps d'un jaune ocré terne. La tête est plus arrondie derrière les yeux que chez maculatus. Le bord cervical à peine plus large. Le scape dépasse d'environ la moitié de sa longueur le bord postérieur de la tête. Le reste comme chez liocnemis Em. 
Rio Mbalé ${ }^{1}$.

Camponotus (Myrmoturba) maculatus F. st. pictiventris Mayr.

Rio Mbalé, 3 ఛ; Cakindo, 1 ఫ.

Camponotus (Myrmosericus) rufoglaucus Jerd. st. controversus Sants.

Chimporo, $\Varangle<\Varangle$. Légère variété avec les pattes moins obscures que chez le type, mais plus foncées que chez chaboti Sants. Cette dernière variété est de Huella Lomba (Benguela).

Camponotus (Myrmosericus) vestitus Sm. pectitus Sants.

Cakindo, 2 ఛ.

Diffère de la variété intuens Forel par les antennes et les tibias entièrement rougeâtres et la tête plus large.

Camponotus (Myrmosericus) vestitus Sm. v. lujai Sants.

Mbalé, ఫ.

Cette variété (in lit.) est très voisine de intuens For., mais n'a pas le devant de la tête rougeâtre et les scapes sont plus longs.

Camponotus (Myrmosericus) vestitus Sm. st. bombycinus n. st.

$\Varangle$ (minor et media). Long.: $5 \mathrm{~mm}, 5$ à $6 \mathrm{~mm}, 5$. Mandibules brun rougeâtre, tout le reste entièrement noir et couvert d'une pubescence longue et dense faisant une pelisse d'un gris argenté. Cette pubescence est beaucoup plus courte et rare sur les appendices. Les segments du gastre sont étroitement bordés de blanc et la pubescence y a la même disposition que chez vestitus Sm. Sur le pronotum elle est concentrique vers une ligne médiane longitudinale. Quelques poils blancs clairsemés se dressent sur le corps et sont moins rares sur et sous la tête.

1 Camponotus (Myrmoturba) maculatus F. st. radamoides For. v. citinus n. var. (fig. $9 c$ ).

ఫ. Long': $13^{\mathrm{mm}}$ à $14^{\mathrm{mm}}$ (major ?). D'un jaune roussâtre plus vif que chez liocnemis et calceatus, avec les tibias et metatarses des deux dernières paires noirs comme chez calceatus et avec la même disposition des couleurs sur la tête et le thorax. Les taches du gastre sont plus nettement limitées et celles du quatrième tergite beaucoup plus petites et arrondies. Celles du premier tergite ne se rencontrent pas au-dessus de l'articulation pétiolaire ni avec celle du premier sternite comme c'est le cas chez liocnemis et calceatus. Tête mate; les angles postérieurs, le dos du thorax un peu luisants. Gastre luisant. La tête est un peu plus étroite que chez calceatus, longue de $3^{\mathrm{mm}}, 5$, large de $2 \mathrm{~mm}, 8$. Le scape dépasse d'un cinquième environ le bord postérieur de la tête. Tibias comme chez liocnemis.

૪๐". Long.: $8 \mathrm{~mm}$. Ressemble comme couleur à calceatus, mais la moitié postérieure de la tête est du même fond roussâtre que le dos du thorax. Les côtés de la tête, derrière les yeux, sont moins arrondis. Pour le reste comme chez calceatus.

Congo belge: Uere Bili. Musée du Congo à Tervueren. 
Tête allongée comme chez vestitus, les côtés parallèles devant les yeux un peu convergents en arrière de ceux-ci, avec un bord postérieur transversal chez l'ouvrière media et convexe chez la petite ouvrière. Ce bord cache alors un peu le bord cervical. Epistome caréné. Thorax très allongé, médiocrement convexe. La face basale de l'épinotum presque rectiligne est plus de trois fois plus longue que la face déclive, formant toutes deux un angle net très ouvert. L'écaille est épaisse, le sommet arrondi ou en tronquature mousse chez la petite ouvrière. Le gastre est étroit et long. Pattes allongées et comprimées.

Chimporo, $4 \not$.

Race bien distincte par sa belle pelisse couvrant tout le corps. Camponotus (Myrmopiromis) fulvopilosus De Geer. v. flavopilosus Em.

Chimporo, $\not$,, . .

Camponotus (Myrmopsamma) mystaceus Em. v. exsanguis For.

Chimporo, $3 \not{q}$. Un peu plus foncée que exsanguis, surtout les tarses, mais plus claire que le type.

Camponotus (Myrmosaga) schoutedeni For.

Rio Mbalé, 2 ఫे.

Camponotus (Myrmotrema) avius Sants.

Cakindo, $\Varangle<\Varangle$. J'ai décrit cette espèce dans cette Revue en 1926 sur des exemplaires de la Rhodesia du Sud (Arnold).

Camponotus (Myrmotrema) monardi n. sp.

$\Varangle$ major. Long.: $8^{\mathrm{mm}}, 5$. Noire. Mandibules, antennes, trochanters, tibias et tarses rougeâtres. Epistome, front, thorax et abdomen mat, densément ponctués-réticulés. Le reste de la tête luisant, avec une fine réticulation fondamentale. Une ponctuation en fossettes s'étendant jusqu'au bord postérieur de la tête, elle est bien plus dense sur les joues et l'épistome, où le diamètre des points égale presque leurs intervalles, et dont le fond est occupé par un tout petit poil couché. Pilosité dressée raide, obtuse, jaunâtre vers le bout, plus claire vers la base, plus clairsemée sur le thorax que sur le gastre, et bordant la face déclive de l'épinotum et l'écaille. Le gastre est orné d'une pubescence dorée, oblique, aussi longue qu'espacée, avec une bande glabre médiane bien visible sur les deuxième et troisième tergites. Cette pubescence est un peu plus dorée que chez grandidieri, mais un peu moins dense. 
Tète un peu plus étroite que chez grandidieri, les côtés subparallèles dans les deux tiers postérieur chez l’ $\Varangle$ maxima (convergents à partir du tiers postérieurs chez grandidieri). Yeux un peu plus grands et placés à la même hauteur que chez grandidieri, mais leur grand diamètre est dirigé obliquement en bas et en dehors au lieu d'être parallèle au bord latéral. Ocelle frontal plus ou moins développé (présent chez les deux exemplaires examinés). Sillon frontal plus court que les arêtes frontales qui sont aussi écartées derrière que longues. Aire frontale très petite. Epistome rectangulaire, peu convexe, sans carène, à bord antérieur faiblement arqué. Mandibules convexes, sculptées comme l'épistome, armées de 6 dents. Le scape cylindrique dépasse d'environ son épaisseur le bord postérieur de la tête. Le profil de la face basale de l'épinotum et du mésonotum est presque horizontal et celui de la face déclive presque vertical, avec l'angle brèvement arrondi. La face basale est d'un bout à l'autre également et transversalement convexe (en coupe d'arc elliptique). Sutures dorsales bien imprimées. Ecaille à sommet ovale et presque tranchant, la face antérieure un peu convexe et la face postérieure plane.

Angola, Planalto, Lobito, III, 1929. $3 \Varangle$ dont l'une sans tête.

Espèce voisine de grandidieri For. par sa couleur et ses fossettes, mais distincte par sa tête en grande partie luisante, ses yeux obliques, son ocelle et son thorax plus robuste. Forel a décrit sommairement une $q$ unique de l'Angola sous le nom de $C$. auropubens var., argentopubens, dont la partie postérieure de la tête est aussi luisante mais dont ses fossettes sont comme chez auropubens, c'est-à-dire tout à fait confluentes, avec de grands poils. Chez le Camp. benguelensis Sants. le thorax fait passage à celui de monardi, mais la tête mate, les yeux à peine obliques le rapprochent plutôt de grandidieri.

Camponotus (Myrmopelta) barbarossa Em.

Timbolé, $1 \not{q}$.

Camponotus (Myrmopelta) chrysurus Gerst.

Chimporo, 1 ๖..

Camponotus (Myrmopelta) chrysurus Gerst. st. apelis For.

Kimbolé, 5 q. La grande $\Varangle$ a lạ tête allongée et l'épistome silloné au milieu. 
Camponotus (Myrmopelta) vividus Sm. v. reginae For. (fig. 10, a, b).

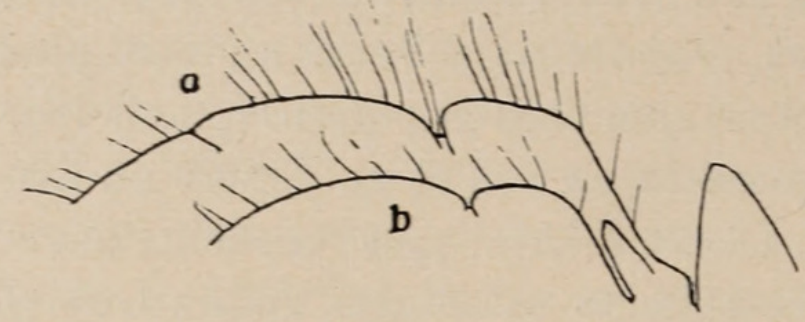

FIG. 10.

Camponotus (Myrmopelta) vividus $\mathrm{Sm}$. v. reginae For.

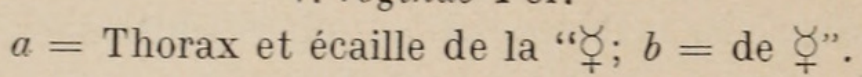

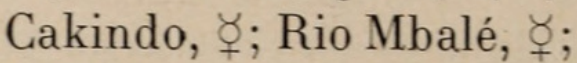
Chimporo, $\not{q}<\not{q}$.

Ainsi que le montre la figure, l'échancrure métanotale de la grande $\Varangle$ est relativement profonde, plus même que chez vividustype ${ }^{1}$. Camponotus (Orthonotomyrmex) mayri For. v. chimporensis $\mathrm{n}$. var.

ఛ. Long.: $7 \mathrm{~mm}$ à $11 \mathrm{~mm}$. Noire. Mandibules et base des scapes presque aussi foncés que le reste de l'article. Pilosité dressée et pubescence un peu plus faible que chez le type. Epinotum et pétiole pas plus fortement sculptés (beaucoup plus fortement chez cubangensis For.). Diffère surtout par la face basale de l'épinotum moins arquée et nettement plus longue, presque aussi longue que le mésonotum (bien plus courte chez mayri). Cette variété fait passage à la race sankisianus For.

Chimporo, $3 \underset{\wp}{\text {; }}$ Cakindo, 1 ఛ.

Camponotus (Orthonotomyrmex) mayri For. st. sankisianus For.

Rio Mbalé, 3 ఛ.

Camponotus (Orthonotomyrmex) cubangensis (Forel).

(Syn.: C. mayri, v. cubangensis. Forel, 1901, Mitt. Schweiz. Ent. Ges. X., p. 309.)

ఛ. Long.: $6 \mathrm{~mm}^{\mathrm{m}}, 5$ à $10 \mathrm{~mm}$. Cette forme est bien distincte du C. mayri par son épinotum convexe, non bordé, grossièrement ridéréticulé ainsi que le pétiole. Il est très voisin du $C$. dofleini For. dont il diffère par la couleur roussâtre de la base du scape, le bord postérieur de l'épinotum un peu plus prolongé derrière, surtout. chez les $\Varangle$ minor. Les denticules de l'épistome, signalés par Arnold, ne sont pas constants. Le bord est orné de gros poils espacés et

1 Les figures que j'ai désignées comme reginae (SAntschi, 1926, Ann. Soc. Ent. France, p. 18, fig. 1, E, F) se rapportent en réalité à une forme de petite taille de la v. meinerti For. (4 $\mathrm{mm}, 2$ à $8 \mathrm{~mm})$, de Brazzaville, qui ne mérite pas de nom. En outre, des signes " $\varnothing^{\prime}(=\varnothing$ major $)$ et $\varnothing "\left(=\varnothing{ }^{\prime}\right.$ minor $)$ ont été en partie intervertis dans la légende des figures des variétés du $C$. vioidus, p. 19. Il faut lire: " $\Varangle$ pour les lettres B, C, E, G, et $\measuredangle$ " pour les lettres A, D, F, H. 
partant chacun d'une fossette séparée de la voisine par un prolongement angulaire plus ou moins net.

Les Camponotus dofleini et ledieui Forel me paraissent être que de simples variétés du cubangensis. Monsieur le Dr J. CarL, qui a eu la bonté d'examiner pour moi les types dofleini et cubangensis, m'écrit que le premier a le devant de l'épinotum moins fortement sculpté. Chimporo, 3 ఛ.

Camponotus (Orthonotomyrmex) sericeus F. v. sulgeri Sants.

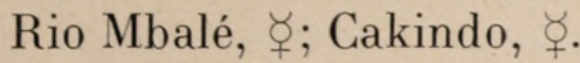

Polyrhachis (Myrma) schistacea Mayr.

Timbolé, 6 ఛ.

Polyrhachis (Myrma) schistacea Mayr st. atrociliata Sants. v. benguelensis Sants.

Chimporo, 8 ఛ; Timbolé, ఫ. 


\section{INDEX BIBLIOGRAPHIQUE}

1915-1922. Arnold, G. A monograph of the Formicidae of South Africa. Ann. of South African Mus., XIV.

1926. — Id. Appendix. Ibid. Vol. XXIII.

1910-1925. Emery. Formicidae. In: Genera Insectorum fase. 102, 137, 174, 183.

1886. Forel, A. Etudes myrmécologiques. Ann. Soc. Ent. Belgique, XXX, p. 131-215.

1894. - Abessinische und andere afrikanische Ameisen. Mitt.Schweiz. Ent. Ges., IX, p. 64-100.

1901. - Einige neue Ameisen aus Südbrasilien, Java, Natal und Mossamedes. Ibid., X, p. 304-311.

1909. - Fourmis de Benguela récoltées par M. Crigton Wellman. Ann. Soc. Ent. Belgique, LIII, p. 64-69.

1910. - Notes sur quelques Fourmis d'Afrique. Ibid., LIV, p. 421458.

1922. — Glanures myrmécologiques. Rev. Suisse de Zool., XXX, p. $87-102$.

1911. Santschi, F. Nouvelles Fourmis du Congo et du Benguela. Rev. Zool. Afr., I, p. 204-217.

1913. - Glanures de Fourmis africaines. Ann. Soc. Ent. Belgique, LVII, p. 311-314.

1914. - Formicides de l'Afrique occidentale et anstrale. Voyage de M. le prof. Silvestri. Boll. Lab. Zool. gén. e agr. Portici, VIII, p. 311-385.

1915. Nouvelles Fourmis d'Afrique. Ann. Soc. Ent. France, LXXXIV, p. 244-282.

1925. - Mission de Rohan-Chabot en Angola et Rhodesia, 1912-1914, IV. Formicides, p. 159-188.

1922. Wheeler, W. M. Ants of the American Museum Congo Expedition. A contribution to the Myrmecology of Africa. Bull. Amer. Mus. of Nat. Hist., XLV. 


\section{$2 \mathrm{BHL}$ Biodiversity Heritage Library}

Santschi, Félix. 1930. "Formicides de l'Angola." Revue suisse de zoologie 37, 53-82. https://doi.org/10.5962/bhl.part.117942.

View This Item Online: https://www.biodiversitylibrary.org/item/148561

DOI: https://doi.org/10.5962/bhl.part.117942

Permalink: https://www.biodiversitylibrary.org/partpdf/117942

\section{Holding Institution}

American Museum of Natural History Library

\section{Sponsored by}

BHL-SIL-FEDLINK

\section{Copyright \& Reuse}

Copyright Status: In copyright. Digitized with the permission of the rights holder.

Rights Holder: Muséum d'histoire naturelle - Ville de Genève

This document was created from content at the Biodiversity Heritage Library, the world's largest open access digital library for biodiversity literature and archives. Visit BHL at https://www.biodiversitylibrary.org. 